\title{
Optically pumped ultraviolet lasing from nitride nanopillars at room temperature
}

\author{
Rui Chen, ${ }^{1}$ H. D. Sun, ${ }^{1, a)}$ T. Wang, ${ }^{2}$ K. N. Hui, ${ }^{3}$ and H. W. Choi ${ }^{3, a)}$ \\ ${ }^{1}$ Division of Physics and Applied Physics, School of Physical and Mathematical Sciences, \\ Nanyang Technological University, Singapore 637371, Singapore \\ ${ }^{2}$ Department of Electronic and Electrical Engineering, EPSRC National Centre for III-V Technologies, \\ University of Sheffield, Sheffield S1 3JD, United Kingdom \\ ${ }^{3}$ Department of Electrical and Electronic Engineering, Semiconductor Lighting and Display Laboratory, \\ The University of Hong Kong, Hong Kong
}

(Received 24 March 2010; accepted 5 April 2010; published online 14 June 2010)

\begin{abstract}
A vertical cavity structure composing of an in situ grown bottom $\mathrm{Al}_{\mathrm{x}} \mathrm{Ga}_{1-\mathrm{x}} \mathrm{N} / \mathrm{Al}_{\mathrm{y}} \mathrm{Ga}_{1-\mathrm{y}} \mathrm{N}$ distributed Bragg reflector and a top $\mathrm{SiO}_{2} / \mathrm{HfO}_{2}$ dielectric mirror for ultraviolet (UV) emission has been demonstrated. Close-packed nanopillars with diameters of around $500 \mathrm{~nm}$ have been achieved by the route of nanosphere lithography combined with inductively-coupled plasma etching. Optically-pumped UV lasing at a wavelength of $343.7 \mathrm{~nm}(3.608 \mathrm{eV})$ was observed at room temperature, with a threshold excitation density of $0.52 \mathrm{MW} / \mathrm{cm}^{2}$. The mechanism of the lasing action is discussed in detail. Our investigation indicates promising possibilities in nitride-based resonant cavity devices, particularly toward realizing the UV nitride-based vertical-cavity surface-emitting laser. (C) 2010 American Institute of Physics. [doi:10.1063/1.3449576]
\end{abstract}

Light-emitting diode (LED) and laser device based on group III nitride (III-N) have drawn intensive research interest and achieved impressive progresses in the last decade. ${ }^{1-8}$ Enormous research activities are currently focusing on the realization of ultraviolet (UV) LEDs and sensors. ${ }^{2,9}$ However, the lack of a viable approach for the fabrication of UV nitride-based vertical-cavity surface-emitting lasers (VCSELs) represent a significant shortcoming in III-N device technology. One essential requirement for producing this kind of laser is that photonic confinement must be achieved, thus the fabrication of high-quality distributed Bragg reflectors (DBRs) is essential. Our previous research work has demonstrated in-situ growth of high-quality crack-free $\mathrm{AlGaN}$ DBRs with peak reflectivity around $350 \mathrm{~nm} .{ }^{10}$ More recently, the fabrication of AlGaN DBR for AlInGaN active material has been reported, and a large enhancement in the performance of UV-LEDs with emission wavelength below the bandgap of $\mathrm{GaN}(362 \mathrm{~nm})$ have been demonstrated. ${ }^{11}$ The employment of DBRs for AlInGaN active material not only improves the optical extraction efficiency, but also enables the possibility of UV nitride-based vertical cavity. However, up till now, the UV nitride-based vertical cavity emitter is rarely reported, especially for nanopillars structures which can provide three-dimensional (3D) confinement of light on the scale of photon wavelength. ${ }^{12}$ This 3D confinement of light will introduce cavity electrodynamics effects which largely enhance the corresponding coupling strength. ${ }^{13}$

Common nanostructure fabrication processes which have been used over the past few years include electronbeam lithography (EBL) and x-ray lithography (XRL). However, the serial patterning natures of these approaches make fabrication costly and inefficient. In contrast, nanosphere lithography (NSL) has excelled as an inexpensive, material specific and high-throughput nanostructure fabrication

${ }^{a)}$ Electronic addresses: hdsun@ntu.edu.sg and hwchoi@eee.hku.hk. process. ${ }^{14}$ In this paper, we demonstrate the fabrication of close-packed nitride-based nanopillars using the NSL method. The sample shows UV lasing from the AlInGaN active material under optical pulse excitation with a threshold excitation density of $0.52 \mathrm{MW} / \mathrm{cm}^{2}$ at room temperature. The effect of the vertical cavity and the mechanism of lasing are discussed in detail.

The sample used herein was a UV-LED wafer grown on c-plane sapphire substrates by a low-pressure metal-organicchemical-vapor-deposition (MOCVD). The bottom DBRs were formed by 25 pairs of $36.5 \mathrm{~nm} \mathrm{Al}_{0.49} \mathrm{Ga}_{0.51} \mathrm{~N}$ and $34.5 \mathrm{~nm} \mathrm{Al}_{0.16} \mathrm{Ga}_{0.84} \mathrm{~N}$ layers. The active region consists of 10 pairs of $2.5 \mathrm{~nm} \mathrm{Al}_{0.05} \mathrm{In}_{0.045} \mathrm{Ga}_{0.905} \mathrm{~N}$ and $5.0 \mathrm{~nm}$ $\mathrm{Al}_{0.2} \mathrm{In}_{0.002} \mathrm{Ga}_{0.798} \mathrm{~N}$ quaternary multi-quantum wells (MQWs), which are sandwiched by an n-type and p-type AlGaN/GaN superlattices, respectively. Details of the structure fabrication and device characterization can be found in Refs. 10 and 11 . The microcavity was completed by deposition of a four-period $\mathrm{SiO}_{2} / \mathrm{HfO}_{2}$ DBRs on the top of the wafer by radio frequency (RF) magnetron sputtering. The $\mathrm{SiO}_{2}$ layers was sputtered using single $\mathrm{SiO}_{2}$ target in an $\mathrm{Ar}$ ambient, while the $\mathrm{HfO}_{2}$ layers was sputtering using $\mathrm{Hf}$ metal target in an $\mathrm{O}_{2}$ ambient. The morphology of the nanostructured sample was determined by field-emission scanning electron microscope (FESEM). The photoluminescence (PL) measurements were performed at room temperature with a pulsed Nd:YAG $4^{\text {th }}$ harmonic $(266 \mathrm{~nm})$ laser as an excitation source and the signal was dispersed by a $750 \mathrm{~mm}$ monochromator combined with suitable filters, and detected by an UV-enhanced charged coupled device (CCD). The pulse width and repetition rate of the pulse laser are $1.0 \mathrm{~ns}$ and $60 \mathrm{~Hz}$, respectively.

Figures 1(a)-1(d) present schematic diagrams of the nanopillars fabrication process flow. The sample was initially coated with plain silica $\left(\mathrm{SiO}_{2}\right)$ nanospheres (having mean diameters of $500 \mathrm{~nm}$ with a uniformity of better than $1 \%$ acquired from Corpuscular Inc.) to form a hexagonally 

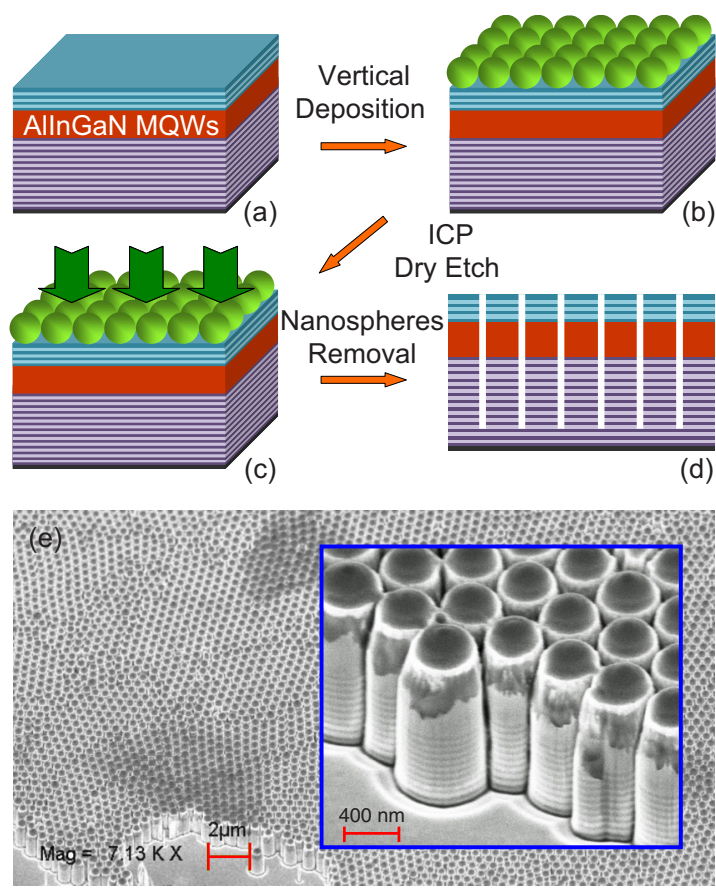

FIG. 1. (Color online) [(a)-(d)] Schematic diagrams depicting the fabrication process of the nitride-based nanopillars. (e) FESEM image showing nanopillars array across the nitride sample. In the close-up image shown in the inset of (e), nanopillars of high aspect ratio can be clearly resolved.

closed-packed (HCP) monolayer pattern on the surface by spin-coating. Subsequently, the sample was subjected to highly selective inductively-coupled plasma (ICP) dry etching for pattern transfer using a gas chemistry of $\mathrm{Cl}_{2}$ and $\mathrm{Ar}$, during which the monolayer of nanospheres served as a hard mask. Finally, the nitride-based nanopillars were formed and the $\mathrm{SiO}_{2}$ nanospheres were removed by sonication in deionized water. ${ }^{15}$ Figure 1(e) shows the FESEM image of the completed sample; highly-ordered closed-packed nanopillars over large areas can be observed. From the close-up FESEM image in the inset of Fig. 1(e), high aspect ratio nanopillars can be seen, with the AlGaN DBR clearly resolved. It is found that the height of the nanopillars is about $1 \mu \mathrm{m}$ and the mean diameter is around $500 \mathrm{~nm}$.

The optical spectra of the samples were recorded in a back scattering geometry with a beam incident angle of $45^{\circ}$ and the emission collected in the direction perpendicular to the sample surface. The PL spectra of the as-grown and nanopillar samples are shown in Fig. 2. It is noted the asgrown sample exhibits a strong emission peak located at around $336 \mathrm{~nm}$, corresponding to the recombination in the AlInGaN MQWs. It was found that the ratio between the relatively PL intensities after and before etching is around $75 \%$. However, the emission peak of the nanopillars is similar with the as-grown chip under same laser excitation, which reveals that the sample is not damaged during the nanopillars formation processes.

Figure 3 shows the PL spectra of the nanopillar sample under different excitation densities at room temperature. It is observed that under low excitation such as $0.34 \mathrm{MW} / \mathrm{cm}^{2}$, the spectrum displays a weak spontaneous emission band from the AlInGaN MWQs. When the excitation density raised to $0.52 \mathrm{MW} / \mathrm{cm}^{2}$, a sharp peak located at $343.7 \mathrm{~nm}$ $(\sim 3.608 \mathrm{eV})$ emerges from the emission spectrum, the intensity of which increases rapidly with the further raising of

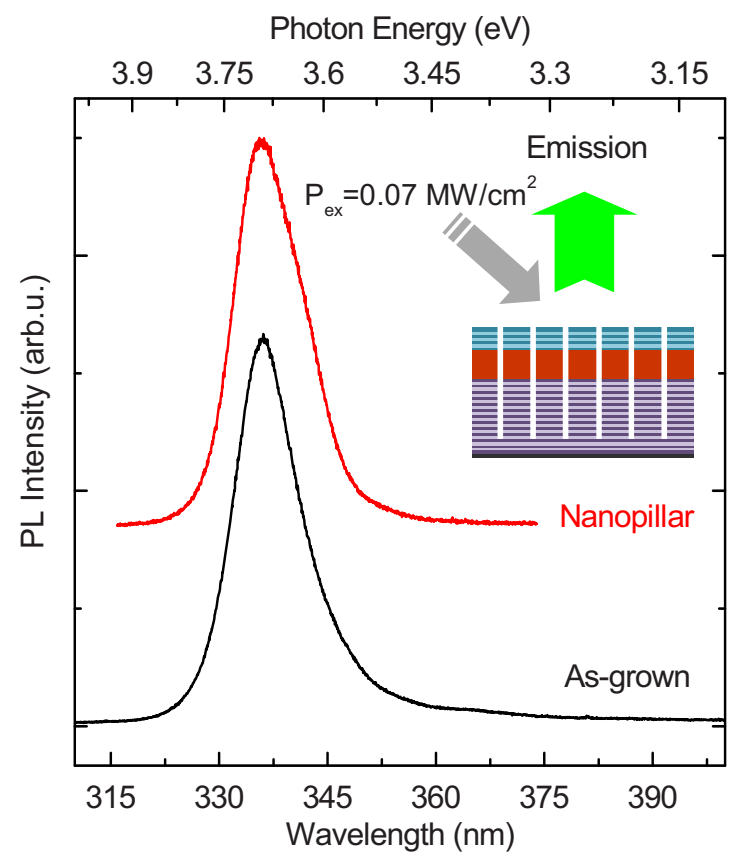

FIG. 2. (Color online) PL spectra of the as-groan and nanopillar sample taken at room temperature. The curves are normalized and shifted in the $y$-axis for better comparison.

excitation density. A plot of integrated PL intensity of this peak with excitation density is shown in the inset of Fig. 3 . Such a nonlinear increase of the emission integrated intensity is a characteristic of stimulated emission. In comparison, the integrated PL intensity of the broad emission from AlInGaN MQWs demonstrates a linear relationship with the excitation density (not shown), which further confirms stimulated emission from the nanopillar sample. The FWHM of the sharp peak is around $22.5 \mathrm{meV}$ under excitation density of $1.14 \mathrm{MW} / \mathrm{cm}^{2}$, thus the quality $(Q)$ factor is estimated to be around 160 according to the deification $Q=E / \Delta E$, where $E$ is the peak energy and $\Delta E$ is the FWHM of the peak, respectively.

The resonant wavelength $\lambda$ and $Q$ factor of a FabryPérot (F-P) cavity are determined by the equations, $2 n L$ $=m \lambda$ and $Q=2 \pi n L / \lambda(1-R)$, respectively, where $L$ is the cavity length which is equal to the distance between the epitaxial and dielectric DBRs $(L=595 \mathrm{~nm}),{ }^{11} n \approx 2.3$ is the mean refractive index of the cavity region $L,{ }^{16} \mathrm{~m}$ is an inte-

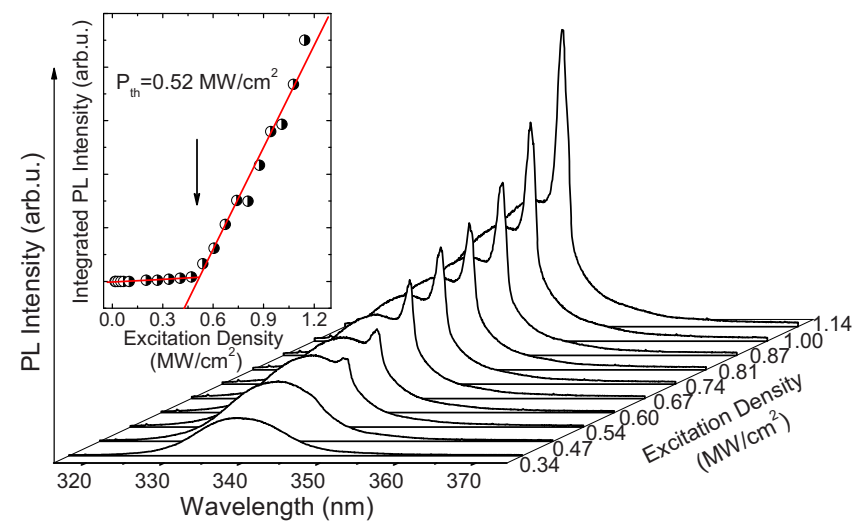

FIG. 3. (Color online) The emission spectra under different excitation density for the nanopillar sample. Figure in the inset shows the dependence of the PL integrated intensity on the laser excitation density. 


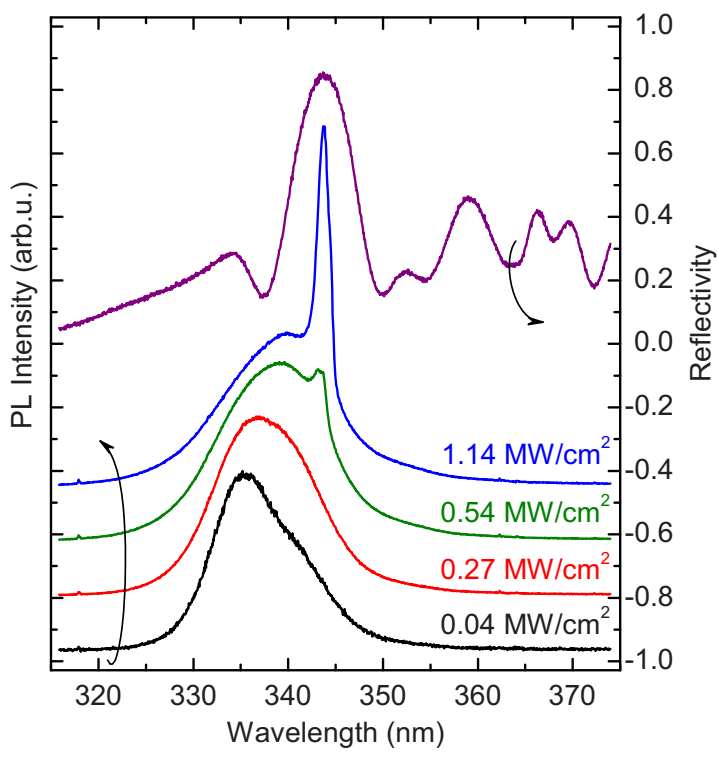

FIG. 4. (Color online) The reflectance spectra of the nanopillar sample together with the emission spectra under different excitation densities.

ger, $\lambda$ is the resonant wavelength, and $R$ is the reflectivity at the active region/DBRs boundary $(R \approx 86 \%){ }^{10}$ From the equation given above, the resonant wavelength is determined to be $342.1 \mathrm{~nm}$ corresponding to $m=8$, and the $Q$ factor is deduced to be 179.5 at $342.1 \mathrm{~nm}$. The observed $Q$ factor is relatively low and the reason should be attributed to optical scattering due to the roughness of the nanopillars' sidewall which enhanced the contributions of intrinsic and extrinsic photon loss channels. ${ }^{17}$ The simulated resonant wavelength $(342.1 \mathrm{~nm})$ is very close to the observed value $(343.7 \mathrm{~nm})$ from our measurement. The mode spacing between the closest longitudinal modes is expected to be $42.8 \mathrm{~nm}$ according to the equation $\Delta \lambda=\lambda^{2} / 2 n L$. Only a single F-P mode is allowed in the entire range of measured PL energies (320-370 $\mathrm{nm}$ ) from the AlInGaN MQWs due to the large mode spacing. It is noted that all the discussions above support the F-P lasing action. Consequently, we attribute the observed lasing to the vertical cavity formed by epitaxial and dielectric DBRs.

In order to further investigate the mechanism of the lasing, the reflectance measurement of the nanopillar sample is carried out. Figure 4 displays the reflectivity of the nanopillar sample together with selected PL spectra under different excitation density. The as-grown sample used here is a UVLED wafer. Although the position of the AlInGaN MQWs is not well designed to be located at the antinodes of the standing wave formed inside the cavity, the top four-period dielectric DBR provides a broad stop-band, so the reflectance of the nanopillar sample is similar to that of the as-grown. As depicted in Fig. 4, the nanostructured sample demonstrates a flat-topped stop-band between 342 and $345 \mathrm{~nm}$. Owing to the high reflectively, stimulated emission can be achieved with low threshold energy due to superior optical confinement. It is interesting to note that the F-P cavity mode located exactly in this region, and thus the UV lasing from the nanopillars can be observed. Although the quality factor of the cavity is compromised by the fabrication of nanopillars, it provides a proof-of-concept demonstration of UV lasing in vertical microcavity structures with dimensions as small as $500 \mathrm{~nm}$ in diameter. In order to fully implement vertical resonate cavity device, improvement of the cavity quality and full structure design are needed, and research down this line points toward the realization of the elusive UV nitride-based VCSEL.

In conclusion, we have fabricated close-packed nitridebased nanopillars with diameter around $500 \mathrm{~nm}$ by NSL. The optical properties of the samples were investigated at room temperature under pulse laser excitation. UV lasing was observed with a threshold of $0.52 \mathrm{MW} / \mathrm{cm}^{2}$ of the excitation density and the observation was attributed to the vertical cavity formed by embedded semiconductor DBRs and the top dielectric mirror.

H.D.S. acknowledges support from Singapore MOE under Grant No. RG40/07. This work was partially supported by a GRF grant of the Research Grant Council of Hong Kong (Project No. HKU 7118/09E).

${ }^{1}$ T. Someya, R. Werner, A. Forchel, M. Catalano, R. Cingolani, and Y. Arakawa, Science 285, 1905 (1999).

${ }^{2}$ M. Asif Khan, M. Shatalov, H. P. Maruska, H. M. Wang, and E. Kuokstis, Jpn. J. Appl. Phys., Part 1 44, 7191 (2005).

${ }^{3}$ Y. Inoue, T. Hoshino, S. Takeda, K. Ishino, A. Ishida, H. Fujiyasu, H. Kominami, H. Mimura, Y. Nakanishi, and S. Sakakibara, Appl. Phys. Lett. 85, 2340 (2004).

${ }^{4}$ S. Keller, C. Schaake, N. A. Fichtenbaum, C. J. Neufeld, Y. Wu, K. McGroddy, A. David, S. P. DenBaars, C. Weisbuch, J. S. Speck, and U. K. Mishra, J. Appl. Phys. 100, 054314 (2006).

${ }^{5}$ M. Diagne, Y. He, H. Zhou, E. Makarona, A. V. Nurmikko, J. Han, K. E. Waldrip, J. J. Figiel, T. Takeuchi, and M. Krames, Appl. Phys. Lett. 79, 3720 (2001).

${ }^{6}$ H. W. Choi, K. N. Hui, P. T. Lai, P. Chen, X. H. Zhang, S. Tripathy, J. H. Teng, and S. J. Chua, Appl. Phys. Lett. 89, 211101 (2006).

${ }^{7}$ L. E. Cai, J. Y. Zhang, B. P. Zhang, S. Q. Li, D. X. Wang, J. Z. Shang, F. Lin, K. C. Lin, J. Z. Yu, and Q. M. Wang, Electron. Lett. 44, 972 (2008).

${ }^{8}$ J. Y. Zhang, L. E. Cai, B. P. Zhang, S. Q. Li, F. Lin, J. Z. Shang, D. X. Wang, K. C. Lin, J. Z. Yu, and Q. M. Wang, Appl. Phys. Lett. 93, 191118 (2008).

${ }^{9}$ T. Onuma, S. Keller, S. P. DenBaars, J. S. Speck, S. Nakamura, U. K. Mishra, T. Sota, and S. F. Chichibu, Appl. Phys. Lett. 88, 111912 (2006).

${ }^{10}$ T. Wang, R. J. Lynch, P. J. Parbrook, R. Butte, A. Alyamani, D. Sanvitto, D. M. Whittaker, and M. S. Skolnick, Appl. Phys. Lett. 85, 43 (2004).

${ }^{11}$ T. Wang, P. J. Parbrook, C. N. Harrison, J. P. Ao, and Y. Ohno, J. Cryst. Growth 267, 583 (2004).

${ }^{12}$ H. Lohmeyer, K. Sebald, C. Kruse, R. Kroger, J. Gutowski, D. Hommel, J. Wiersig, N. Baer, and F. Jahnke, Appl. Phys. Lett. 88, 051101 (2006).

${ }^{13}$ S. Reitzenstein and A. Forchel, J. Phys. D: Appl. Phys. 43, 033001 (2010)

${ }^{14}$ C. L. Haynes and R. P. Van Duyne, J. Phys. Chem. B 105, 5599 (2001).

${ }^{15}$ W. N. Ng, C. H. Leung, P. T. Lai, and H. W. Choi, Nanotechnology 19, 255302 (2008).

${ }^{16}$ G. M. Laws, E. C. Larkins, I. Harrison, C. Molloy, and D. Somerford, J. Appl. Phys. 89, 1108 (2001).

${ }^{17}$ T. Rivera, J. P. Debray, J. M. Gerard, B. Legrand, L. Manin-Ferlazzo, and J. L. Oudar, Appl. Phys. Lett. 74, 911 (1999). 